\title{
Current issues and forthcoming events
}

\author{
Edited by G. Hussein Rassool
}

\section{IN TER NATIONAL}

\section{SARS (severe acute respiratory syndrome)}

Severe acute respiratory syndrome (SARS) is a respiratory illness that has recently been reported in Asia, North America, and Europe. SARS, an atypical pneumonia of unknown aetiology, has killed more than 200 people worldwide, and infected almost 4000.

SARS was first recognized on 26 February 2003 in Hanoi, Vietnam. A man (index case) was admitted to hospital in Hanoi with a high fever, dry cough, myalgia and mild sore throat. Over the next four days he developed increasing breathing difficulties, severe thrombocytopenia, and signs of Adult Respiratory Distress Syndrome and required ventilator support. Despite intensive therapy he died on the 13 March after being transferred to Hong Kong Special Administrative Region of China. On 5 March, seven health care workers who had cared for the index case also became ill (high fever, myalgia, headache and less often sore throat). The onset of illness ranged from 4 to 7 days after admission of the index case.

SARS is currently believed to be caused by a new coronavirus strain that is spread by close person-to-person contact. Symptoms of SARS typically begin with a fever greater than $100 \cdot 4^{\circ} \mathrm{F}$ $\left[>38.0^{\circ} \mathrm{C}\right]$, headache, an overall feeling of discomfort, body aches, and mild respiratory symptoms. After 3-7 days, a lower respiratory phase begins with the onset of a dry, non-productive cough or dyspnoea, which might be accompanied by or progress to hypoxaemia.

Healthcare workers have been identified as a primary 'at risk' group for exposure to SARS. Caring for and treating persons with SARS exposes individuals to infectious droplets. Possible ways SARS can be transmitted include touching people's skin or objects contaminated with infectious droplets, then touching your own eyes, nose, or mouth, or through airborne inhalation of aerosolized droplets. The Centers for Disease Control and Prevention (CDC), USA, reports that 'transmission to health care workers appears to have occurred after close contact with symptomatic individuals (e.g. persons with fever or respiratory symptoms) before recommended infection control precautions for SARS were implemented (i.e. unprotected exposures).' There is also a possibility that the causative agent can remain viable for extended periods of time after drying on environmental surfaces.

The risk of transmission of SARS increases when caring for patients in the absence of appropriate infection control precautions. Instituting proper infection control measures can significantly reduce the risk of exposure and prevent the development of SARS among health care workers. As a primary step in infection control, screening and early identification of suspected SARS cases is essential. Prompt and appropriate isolation of patients suspected of having SARS should also be done. At a minimum, health care workers should use the following precautions:

- standard precautions

- hand hygiene

- eye protection for all patient contact.

\section{Airborne precautions}

- isolation room with negative pressure relative to the surrounding area

- use of a filtering disposable respirator (if unavailable, health care personnel evaluating and caring for suspect SARS patients should wear a surgical mask)

- proper fit-testing respiratory protection is essential

- higher level respiratory protection should be considered, especially for aerosol-generating procedures.
Contact precautions

- gown and gloves for contact with the patient or their environment.

The risk of transmission of SARS can be minimized if the correct precautions are taken. In primary care this means from first contact with a patient who may fit the case definition of suspect or probable SARS. Primary care colleagues should particularly note the need for hygiene and decontamination of the clinical area in which a patient has been assessed prior to referral to hospital.

\section{For more information:}

For updated clinical case definitions, visit:

- Centers for Disease Control and Prevention (CDC) Case Definition: http:// www.cdc.gov/ncidod/sars/casedefinition.htm

- World Health Organization Case Definition: http://www.who.int/csr/ sars/casedefinition/en/

- For general information about SARS: http://www.cdc.gov/ncidod/sars/

For a list of specific Infection Control Guidelines for health care settings visit:

- http://www.cdc.gov/ncidod/sars/infectioncontrol.htm;

- http://www.cdc.gov/ncidod/sars/cleaningpatientenviro.htm

- http://www.phls.co.uk/topics_az/SARS/ management_guidance.htm

\section{Nurses caring for patients in the SARS hit areas saluted by the International Council of Nurses}

On the occasion of International Nurses Day, ICN publicly commended the professionalism and commitment of all nurses, exemplified today by their work caring for SARS patients world-wide. 'The nature of our work means that nurses are in close contact with patients during all phases of their illness, often the first to detect the problem as well as 
to provide consultation, care and follow-up', said Judith Oulton, Chief Executive Officer of the International Council of Nurses (ICN).

'With SARS, as in other crises, nurses work around the clock in difficult circumstances, and with great personal sacrifice. The current public attention focused on SARS highlights the value and critical importance of nurses and their work, everyday and everywhere.' Nurses caring for SARS patients may have to live in or near the health facility where they are working, instead of returning home. Practicing 'barrier nursing' as they must, means wearing layers of protective clothing and tight-fitting masks for long hours and working in isolation from their colleagues. The care they give is essential for the recovery of SARS patients.

For further information contact Linda Carrier-Walker, tel.: +41 22908 0100, fax: +41 22908 0101, E-mail: carrwalk @icn.ch, or visit: http://www.icn.ch

\section{HIV vaccine trial results are an important step forward in developing an effective vaccine, say WHO and UNAIDS}

Preliminary results of a large-scale trial of a candidate AIDS vaccine announced by the US-based biotechnology company VaxGen suggest that it is possible to protect some individuals from HIV infection. The trial of the company's AIDSVAX vaccine appears to show a protective effect among non-Caucasian populations, especially African Americans, although sample sizes were small. However, for the majority of the participants, who were Caucasians, the effect of the vaccine was minimal.

The company stressed, however, that the results only represent findings from an initial analysis. Additional studies will be conducted to further clarify the data. 'These results are promising. The trial provides clear evidence that a vaccine can work', said Dr Peter Piot, Executive Director of UNAIDS. 'However, there is an urgent need for more targeted research to find out why the candidate vaccine only seems to work in certain population subgroups. In the meantime, we must continue to expand existing prevention efforts, which have proved their effectiveness when they are implemented at full scale'.

This trial vaccine is a promising first step, but an effective vaccine providing widespread protection is still not on the horizon. The AIDSVAX Phase III trial was the first large-scale human trial of an HIV vaccine. The trial was made possible because of the involvement of over 5400 volunteers from the United States, Canada, and the Netherlands, the majority of whom were men who have sex with men.

Phase III trials, which involve thousands of volunteers, focus on whether a candidate vaccine is effective and can safely protect people. These are only conducted once Phase I and II trials have ensured that the vaccine is safe and produces an immune response against the virus.

The vaccine used in this trial was designed to reduce susceptibility to infection with HIV subtype B, which is prevalent in the Americas, Western Europe, Australia, and New Zealand. To date, 11 subtypes of HIV-1 have been identified. One of the major challenges in HIV vaccine development is to develop one or multiple vaccines effective against all major subtypes of HIV.

'Continued HIV vaccine research remains an urgent global need', said Dr Gro Harlem Brundtland, DirectorGeneral of WHO. 'We will need many more trials to develop effective HIV vaccines, particularly against the most prevalent HIV subtypes, which are having a devastating impact on populations in sub-Saharan Africa'. Dr Brundtland added that national governments, the private sector and the international community must ensure that a robust programme of vaccine research is continued and that effective prevention, care and treatment programmes are available.

VaxGen is also conducting another Phase III trial in Thailand, involving a vaccine candidate based on HIV subtypes B and E. That trial, which involves more than 2500 volunteers, mostly injecting drug users, is expected to provide additional valuable information about the potential efficacy of this type of candidate vaccine. Results are expected by late 2003. VaxGen is also currently conducting preclinical research to develop a vaccine against the most common subtype, subtype $\mathrm{C}$, which accounts for approximately $50 \%$ of all new HIV infections world-wide.

Several other candidate vaccines based on different HIV subtypes are being tested by other public and private organizations, mostly in the United States and Europe, but also increasingly in developing countries, where a total of 22 vaccine candidates have been or are being tested including in Brazil, Haiti, Kenya, Peru, Thailand, Trinidad and Tobago, and Uganda. At least one of these candidate vaccines is expected to enter Phase III trials this year in Thailand, with results available four years later.

The WHO/UNAIDS HIV Vaccine Initiative was established in 2000 to facilitate the development and evaluation of appropriate HIV vaccines, ensuring that once they are developed they are affordable and available to all people in need. According to UNAIDS and WHO, an estimated 42 million people are living with HIV/AIDS worldwide, of which close to 30 million live in subSaharan Africa. An estimated 5 million people were newly infected with the HIV virus in 2002.

For more information please contact Chris Powell, Information Officer, tel.: (+41 22) 791 2888, E-mail: powellc @who.int, or visit http://www.who.int/

\section{ANA commends congress for securing over \$20 million in additional funding for nurse education programmes}

The American Nurses Association (ANA) have applauded the US Congress for approving $\$ 20$ million in new federal funds for nurse education programmes, including the Nurse Reinvestment Act, as part of the omnibus appropriations bill for fiscal year 2003 .

'We are pleased that Congress recognizes the nursing shortage and the need to invest in recruitment and retention', said ANA President Barbara A. Blakeney. 'These funds will go a long way toward assisting nursing as a profession and will especially help implement the important programmes authorized by the Nurse Reinvestment Act.' President Blakeney noted that ANA is particularly pleased with the increases to the Nurse Corps provision of the Nurse 
Reinvestment Act, which will support scholarships and loan repayments for nursing students. Also encouraging, said President Blakeney, are the increases in funding for the Magnet, career ladders, geriatric-training grant and facultydevelopment provisions of the act. 'These funding increases, combined with the increases for already existing Diversity and Nurse Education and Practice programmes, will go far in advancing the nursing profession', President Blakeney noted.

The Nurse Reinvestment Act (PL 107205), which was signed into law in August 2002, expands authority for existing nursing programmes and creates a number of new ones. For example, the new law authorizes scholarships and loan repayments for nursing students who agree to work in shortage areas after they graduate. In addition, the act authorizes public service announcements to promote nursing as a career, loan cancellations for nursing faculty, grants for geriatric nurse education, and grants to encourage nursing best practices, such as those in the American Nurses Credentialing Center Magnet Recognition Program, for excellence in nursing services.

'We thank Senator Mikulski for her diligence and commitment to securing the necessary funding included in this bill, as well as the other members of Congress who supported it', President Blakeney said. 'These new nurse education funds will be instrumental in stemming the nation's growing nursing shortage and in boosting the image of this most worthy and rewarding profession'. ANA is the only full-service professional organization representing the nation's 2.7 million Registered Nurses through its 54 constituent associations. ANA advances the nursing profession by fostering high standards of nursing practice, promoting the economic and general welfare of nurses in the workplace, projecting a positive and realistic view of nursing, and by lobbying the Congress and regulatory agencies on health care issues affecting nurses and the public. For more information visit http://www.ana.org

\section{CIVILIANS PAY IN WAR}

Members of the International Confederation of Midwives, the International
Council of Nurses, the World Confederation for Physical Therapy, the World Dental Federation, and the World Medical Association have joined in saying civilians pay the terrible price for war in death, injury and disease. Speaking with one voice, the international organizations representing a broad spectrum of the world's health professions have spoken out against all armed conflict.

The terrible health consequences of even 'conventional' war are borne overwhelmingly by civilians, with especially catastrophic effects on women and children's physical and mental health. Death and debilitating injury are coupled with threatened access to safe water, sanitation and food, undermining the health of a population and creating circumstances favourable to epidemic diseases. As infrastructure, homes, and communities are destroyed and people seek safety, more families are added to the already huge population of 12 million refugees and 6 million displaced persons worldwide. Precious resources, financial and human, are diverted to war - inevitably at the expense of investment in health, health systems, and education.

According to the World Health Organization, about 35 people are killed every hour as a direct result of armed conflict. In the twentieth century, an estimated 191 million people lost their lives directly or indirectly as a result of armed conflict, the majority civilians.

Because of the overwhelmingly negative effects on health resulting from war, these health professionals oppose any armed conflict and have chosen to speak out at this time. We strongly encourage governments and ruling parties to find non-violent and democratic means to resolve conflicts and bring about peace.

For further information contact Linda Carrier-Walker, International Council of Nurses, tel.: +41 22908 0100, fax: +41 22908 0101, E-mail: carrwalk@ icn.ch, or visit http://www.icn.ch

\section{RESEARCH}

\section{Improving mental health services for ethnic elders}

A new report from the Royal College of Psychiatrists, 'Psychiatric Services for Black and Minority Ethnic Elders', makes a number of recommendations for improving mental health services in the UK for this under-represented group. To date, psychiatric services for them have received little attention, partly because the number of White elders has been so much greater.

The 1991 UK census showed that there were 10 times as many White elders as those from all minority groups, although this figure may change with the 2001 census. The diversity, wide geographical distribution and lack of knowledge about access to services among ethnic elders have led to an underrepresentation of their psychiatric needs.

This situation is compounded by the fact that the younger ethnic minority population frequently does not share the traditional beliefs and expectation of support from the extended family held by their elders. Information available in translated leaflets and posters is not as effective as it could be, for example, by dissemination though general practitioners and other stakeholders. The report recommends that:

- Acute psychiatric services involving assessment and treatment should remain within mainstream psychiatric services, with ethnic awareness and sensitivity emphasized by training staff in culturally sensitive issues.

- Services providing continuing care in the community should be developed specifically for the appropriate user group.

- Efforts could be made to recruit a racial mix of multidisciplinary staff members reflecting the population served. Ethnic elders need appropriate and accessible mental health services.

- A means to share information would be to set up a website on, or linked to, the Royal College of Psychiatrists' site.

- There should be increased involvement and commitment by all interested stakeholders to involve general practitioners and other key players in establishing good practice for this group.

The report comments that a reliable and informative database of good practice available on a website will also offer training opportunities to interested specialist registrars, and could lead to research that would define appropriate services for ethnic elders.

For further information contact Deborah Hart or Thomas Kennedy in 
the External Affairs Department, tel. +44 (0)207235 2351, E-mail: dhart@ rcpsych.ac.uk or tkennedy@rcpsych. ac.uk, or visit http://www.rcpsych.ac. uk/publications/cr/council/cr103.pdf for the full report.

\section{Cancer patients suffering pain unduly}

A new report finds that despite the wide availability of pain drugs, many patients diagnosed with cancer, are under treated for their pain and suffer unnecessarily. Under treatment is the result of poor diagnosis, assessment, and treatment by physicians; coupled with an unfounded fear of the use of controlled drugs such as opioids. The report indicates that the key to better management of cancer pain is improved educational programmes and the development of dedicated pain management teams.

Cancer pain affects over 3 million people in the US and over half a million in the UK alone. This type of pain can have multiple causes but the most commonly seen are those associated with the tumour itself, and/or with conventional cancer treatments such as chemotherapy and surgery. The severity of cancer pain is often dependent on the type of cancer and stage of the disease.

Diagnosis of the pain type and severity are major determinants and the key to success of any treatment for cancer pain. Failure to accurately assess pain can lead to under treatment. One reason for this failure is that assessment of cancer pain is often complicated by the presence of a number of different types of pain, in addition to a variety of painful conditions that can exist alongside the cancer (such as arthritis). The result is that poor diagnosis of pain in cancer patients remains a significant problem, with many physicians finding it difficult to differentiate between the various pain types; and, many underestimating its severity.

Cancer pain experts interviewed for the report believe that in many cases there is a failure by medical schools to sufficiently educate students in the basics of cancer pain management and palliative care. This contributes to a poor understanding of available diagnosis and assessment techniques. This is compounded by a lack of continuing education for medical professionals who are often unaware of newer diagnostic measures and the benefits of using more than one assessment measure prior, during and as follow-up to treatment.

At present there are numerous best practice guidelines that provide an overview of the most appropriate methods of managing and treating cancer pain. Despite the fact that such approaches have been found to be effective in relieving pain in almost $90 \%$ of patients, up to half of physicians do not make use of the guidelines. The most appropriate treatment of cancer pain is the use of non-opioid analgesics such as paracetamol for mild pain, a weak opioid such as tramadol for moderate pain, and a strong opioid such as morphine for severe pain. At each step, it may be appropriate to add an adjuvant-a drug that has a primary indication other than pain but is used to enhance analgesia in specific circumstances or to treat pain that is unresponsive to conventional analgesics such as an antidepressant.

At present, pain physicians use a huge range of drugs; however, in comparison to the US, fewer patients in the UK are treated with opioids suggesting that physicians are still cautious about their use. It was found that $52 \%$ of patients in the US compared to $35 \%$ in the UK are treated with opioids for moderate pain; and $68 \%$ compared to $50 \%$ for severe pain. This is a significant difference and highlights that physicians are often using inappropriate treatments for more severe pain including low dosages for insufficient time periods. This is often a result of unfounded fears over addiction and side-effects of opioids - both by physicians and patients.

Low treatment rates and inappropriate use of available drugs highlight the need for increased education within the medical community. This is a great opportunity for pharmaceutical companies to become involved in awareness programmes, promoting more appropriate treatments, and thereby influencing physician prescribing. This would result in a win-win situation for both patients and pharmaceutical companies.

Experts believe an additional problem is poor communication between cancer physicians (oncologists) and their patients. Physicians do not always inform patients of the likelihood of pain and many patients are still reluctant to report pain due to fears of its significance and attitudes towards treatment, in particular opioids. Poor levels of communication not only exist between patients and physicians but among healthcare professionals, where there is still insufficient communication between physicians, in particular consultants to primary care physicians and this contributes to poor continuity of care. The research for this report has found that there are still few professionals involved in dedicated cancer pain management teams. Governments and health authorities have a major part to play in making cancer pain management a priority and promoting the development of multidisciplinary cancer teams. The benefits of such a team is evident where they have been rolled out for terminally ill patientswith the patient referred to one palliative care specialist who takes responsibility for stabilizing the pain, setting up a treatment regimen, and informing the primary care physician about appropriate treatment in the outpatient setting.

Poor diagnosis, poor assessment, the choice of less appropriate treatments, plus patients and physicians fears about controlled drugs such as morphine all contribute to under treatment of cancer pain. Education of patients is key to changing attitudes and improving communication between patients and physicians. Awareness of the prevalence of pain as a symptom of cancer and the range of possibilities for treatment would aid patient presentation, compliance and ultimately reduce symptoms. Physician education is key to better assessment and treatment. Dedicated pain management teams have a role to play by trickling down ideas to the level of PCP and thus improving prescribing methods and alleviating unfounded fears over the use of controlled substances.

Further details on this are available from Yasmeen Khan at Datamonitor, tel.: $\quad+44(0) 2076757487$, E-mail: ykhan@datamonitor.com

\section{Type 1 diabetes increases stroke risk}

Type 2 diabetes is a known risk factor for stroke as well as for cardiovascular 
disease. But a British cohort study published in the February 2003 issue of Stroke is the first to determine that type 1 diabetes is also a risk factor (Laing et al. 2003). The effect is similar in all ages, and the magnitude of risk is at least as great as with type 2 diabetes.

'The results from this group of patients with type 1 diabetes show that at all ages death from cerebrovascular disease is higher in the patients with diabetes than in the general population', says lead author Susan P. Laing from the Institute of Cancer Research in Surrey, UK.

From 1972 to 1993 , the Diabetes UK Cohort study identified 23751 patients diagnosed with type 1 diabetes before the age of 30 , and it followed them until December 2000, for an average of 17 years.

Of 1437 deaths, 80 were caused by cerebrovascular disease, which accounted for $4 \%$ of all deaths younger than 40 years and $8 \%$ of all deaths older than 40 years. Standardized mortality ratios (SMR) showed that these rates were significantly higher than expected when compared with the general population. SMR was $3 \cdot 1$ (95\% confidence interval [CI], 2.2-4.3) for men and $4 \cdot 4$ (95\% CI, $3 \cdot 1-6 \cdot 0)$ for women.

In subjects aged 20-39 years, the risk of cerebrovascular death was increased more than five-fold in men and seven-fold in women compared with the general population. Separate analysis of haemorrhagic and non-haemorrhagic stroke still revealed a significant increase in mortality from non-haemorrhagic stroke.

The authors conclude that 'at younger ages, the relative risks of cerebrovascular mortality in patients with type 1 diabetes are very high', and that at all ages they are 'still comparable to those of similarly aged patients with type 2 diabetes. 'These observations emphasize the vital need to identify and treat known cardiovascular risk factors in young people with diabetes', said Dr Laing.

Source: http://www.medscape.com

\section{Reference}

Laing S.P., Swerdlow A.J., Carpenter L.M. et al. (2003) Mortality from cerebrovascular disease in a cohort of 23000 patients with insulin-treated diabetes. Stroke 34, 418-421.

\section{New rapid bedside test for plague}

Investigators have developed a rapid bedside test for plague, according to a report in the January issue of The Lancet (Chanteau et al. 2003). The commentator suggests that in addition to being useful in endemic countries, this test will also enhance our preparedness for bioterrorism.

'With this test, the rapid and costeffective diagnosis of bubonic and pneumonic plague could easily be achieved by health workers in remote sites', said lead author Suzanne Chanteau from the Pasteur Institute and the Ministry of Health in Madagascar. 'Use of the test could help to reduce mortality (through rapid and efficient treatment of patients), morbidity (by rapid implementation of preventive measures), and insecticide resistance of fleas (through rational use of expensive insecticides).' The diagnostic test, which takes up to 15 minutes, uses monoclonal antibodies to the F1 antigen of Yersinia pestis. While detecting $\mathrm{F} 1$ antigen at concentrations as low as $0.5 \mathrm{ng} / \mathrm{mL}$, this test also detected $41.66 \%$ more infections than did bacteriological methods, and $31 \%$ more infections than did ELISA. Sensitivity and specificity were both $100 \%$, positive predictive value was $90.6 \%$, and negative predictive value was $86 \cdot 7 \%$.

In an accompanying commentary, David Dennis and May Chu from the Centers for Disease Control and Prevention in Atlanta, Georgia, praise this study for its comprehensive evaluation in health centres in Madagascar where plague is endemic.

'It is anticipated that it will soon be available in plague-endemic areas world- wide, providing opportunities for validations under various conditions', they write. 'In addition, the test...is expected to fill an important need in bioterrorism preparedness and response.

Source: http://www.medscape.com

\section{Reference}

Chanteau S., Rahalison L., Ralafiarisoa L. et al. (2003) Development and testing of a rapid diagnostic test for bubonic and pneumonic plague. Lancet 361, 211-216.

\section{CONFERENCES}

\section{Multiple Sclerosis Study Day for Health Professional with an Interest in MS, UK}

The study day will be held on 10 September 2003, in Penrith, UK. For further information contact Catherine Thornley, tel.: +44 (0)1462 476704, E-mail: catherine.thornley@mstrust.org.uk or visit http://www.mstrust.org.uk/

\section{6th Annual Scientific Meeting of the Gerontological Society of America, USA}

This meeting will be held 21-25 November 2003, in San Diego, USA. For further information contact The Gerontological Society of America, 1030 15th Street NW, Suite 250, Washington DC 20005, USA, tel.: +1 202 842 1275, or visit http://www.geron.org

\section{Myths and Mountains: Evidence Based Practice in Trauma and Orthopaedic Nursing, UK}

This RCN Society of Orthopaedic and Trauma Nursing Annual Conference and Exhibition will be held 4-6 September 2003, North Wales Conference Centre, Llandudno, Wales. For further information visit the RCN website at http://www.rcn.org.uk 